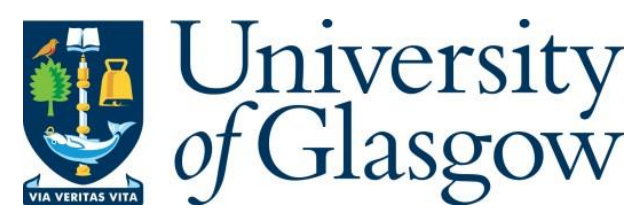

Jamshed, M. A., Brown, T. W.C. and Héliot, F. (2022) Dual antenna coupling manipulation for low SAR smartphone terminals in talk position. IEEE Transactions on Antennas and Propagation, (doi: 10.1109/TAP.2022.3141218).

There may be differences between this version and the published version. You are advised to consult the publisher's version if you wish to cite from it.

https://eprints.gla.ac.uk/262491/

Deposited on: 7 January 2022

Enlighten - Research publications by members of the University of Glasgow https://eprints.gla.ac.uk 


\title{
Dual Antenna Coupling Manipulation for Low SAR Smartphone Terminals in Talk Position
}

\author{
Muhammad Ali Jamshed, Member, IEEE, Tim W.C. Brown, Member, IEEE and Fabien Héliot, Member, IEEE,
}

\begin{abstract}
A rigorous analysis of the concept of coupling manipulation utilizing two antennas suited to modern smartphone devices in talk position for voice calls is presented. By using the optimum relative phase between the elements, they can substantially reduce the specific absorption rate (SAR) but still maintain efficiency due to the splitting of power between them and by exploiting a suitable level of inter element coupling. The same antenna elements can still be used for multiple input multiple output (MIMO) when not in talk position without heavily degrading their fundamental capacity limit but this is of secondary importance. The concept could be applied to frequency ranges used in mobile communications from 1.8 to $6 \mathrm{GHz}$ where the ground plane has sufficient form factor. Extensive simulations using two planar inverted-F antennas (PIFAs) operating at $\mathbf{2 . 4}$ GHz are carried out to demonstrate conceptually how two antennas can be optimized to reduce SAR by over $50 \%$ compared to a single antenna element. SAR reduction is maintained regardless of the user's head composition and how they are handling the device in talk position. Antenna prototypes are measured and compared to verify the capacity when the handset is used away from the body with two MIMO terminal antennas.
\end{abstract}

Index Terms-Coupling manipulation, electromagnetic field (EMF) exposure, specific absorption rate (SAR), multiple input multiple output (MIMO).

\section{INTRODUCTION}

$\mathrm{M}$ OBILE user terminal (UT) devices have evolved in their design and compactness for over three decades. As their usage has relentlessly grown, guidelines were put in place to limit the level of electromagnetic field (EMF) coupled to the human head, quantified by the specific absorption rate (SAR) [1]. In the early days, mobile devices were either in "clam shell" or "candy bar" format and the focus was upon how the external whip antenna could be positioned, or an internal planar antenna could be designed to reduce the SAR by exploiting the ground plane [2]. More recently over the past decade, the widespread usage of the "smartphone" has brought about the need to rethink the way in which the SAR can be substantially reduced for a notably wider, yet thinner, device.

All modern smartphones to date are designed around the multiple input multiple output (MIMO) transceiver technology as it is required by the fourth and fifth generation of cellular

This work has been funded by EPSRC under the grant agreement "EP/P005853/1: Electro-magnetic field exposure reduction/avoidance for the next generations of wireless communication systems". We would also like to acknowledge the support of the University of Surrey, Home of 5G and 6G Innovation Centre (http://www.surrey.ac.uk/5gic) for this work. M. A. Jamshed is with James Watt School of Engineering, University of Glasgow. T. W. C. Brown, and F. Héliot are with the Institute of Communication Systems (ICS), Home of 5G and 6G Innovation Centre, University of Surrey, UK. Email: muhammadali.jamshed@glasgow.ac.uk tim@brown76.name f.heliot@surrey.ac.uk. systems [3] as well as WiFi standards [4]. In general, the multiple antennas in a smartphone device are first and foremost optimized for efficiency and MIMO performance, where the SAR is merely seen as a regulatory constraint and, as such, is often an afterthought of the MIMO antenna design process [5], [6]. Early works related to SAR reduction in UTs have investigated ferrites [7] [8], defected ground structures [9], parasitic elements [10] or metamaterials [11] [12] as ways to decouple the UT antennas from the head, but the already very limited space in a smartphone makes these techniques almost impossible to implement.

The relative phase angle between MIMO antennas has also been identified as a parameter affecting the SAR [13], [14], [15]. More precisely, it has been shown in [13] and [14] that the SAR of a two antenna UT could be significantly reduced by using a suitable relative phase angle between the two antenna elements and [14] proposed a signal processing scheme to implement this finding. In [16] a SAR analysis has been carried out on a variety of UTs with MIMO antennas and showed that the maximum SAR due to relative phase angle between antenna elements can be predicted and, in turn, this can be useful to streamline conformance tests. This work also identified that the SAR does change dramatically with the phase, when considering closely spaced and coupled antennas, and that the power distribution between the antenna elements can affect the SAR, which had earlier been identified in [17]. Other work has also reported that the balance of the power distribution between two elements has some further effect [18]. Though relative phase and power distribution between antenna elements are clear parameters for changing the SAR, the underlying physical principle of this phenomena has not been properly explained and understood. This will enable designing of context aware multiple antennas for mobile terminals that can adapt to low SAR when in talk position using their existing media access control layer.

This paper investigates how two antenna elements on a mobile terminal can be configured to form the lowest possible SAR in talk position, while maintaining efficiency. This is achieved through distribution of power between the elements, the superposition of the fields penetrating into the head but also change in current distribution due to their coupling between them so that relative phase between elements manipulates and reduces the SAR to the human head. It conducts a rigorous analysis of the antenna element requirements, the required element coupling and the impact of changing the inclination angle as well as the position of the earpiece relative to the ear. Through prototyping the antenna, it is verified that the coupling levels are also sufficiently low that MIMO capacity is not substantially compromised when used away from the head. 
However, contrary to conventional MIMO antennas, capacity is a secondary priority while SAR is the first in this work.

The original contributions to knowledge provide a rigorous analysis of how two UT antennas and the ground plane can be utilized to reduce SAR to the human head while not compromising efficiency through optimising the relative phase and coupling between them. Low SAR is maintained regardless of the dielectric composition of the head, the UTs inclination angle or the position the earpiece of relative to the ear hole. Hand grip effects will only increase SAR to the hand as opposed to the head.

The remainder of the paper is organized as follows: Section II discusses the underlying theory behind antenna inter element coupling and change in SAR. This leads to the requirement for exhaustive simulations reported in Section III to evaluate change in SAR and efficiency due to relative phase from two coupled planar inverted-F antenna (PIFA) elements. Section IV analyzes the SAR based on power splitting, field penetration and current distributions. Section V investigates the robustness of the concept regarding inclination angle, position and hand grip. Section VI provides experimental results to verify the impact on MIMO capacity when not used in talk position. The conclusion of the paper is drawn in Section VII.

\section{DUAL ANTENNA COUPLING AND SAR REDUCTION}

Two antenna elements on a handset can reduce the SAR first by splitting the total power halfway into each one. Secondly, optimising the relative phase between elements allows SAR to reduce further due to how the penetrated fields superimpose within the head near the surface. Thirdly, by exploiting the mutual coupling between elements, the relative phase between them alters the current distribution, fields coupled into the head and likewise SAR. The theory behind these three principles are discussed in this section and analysed in this paper.

The mutual coupling refers to an exchange of electromagnetic energy between any two antenna elements placed nearby to each other [19], which is quantified by the scattering parameter $S_{12}$ (or $S_{21}$ ) that relates to the admittance parameters as follows [20]:

$$
S_{12}=\frac{-2 Y_{12} Z_{0}^{2}}{\left(1+Y_{11} Z_{0}\right)\left(1+Y_{22} Z_{0}\right)-Y_{12} Y_{21} Z_{0}^{2}}
$$

where $Z_{0}$ is the characteristic impedance, $Y_{11}$ and $Y_{22}$ are the self admittance at ports 1 and 2 while from the mutual admittances, $Y_{12}=Y_{21}$ when assuming reciprocity. By choosing $Y_{12}$, it can be related to the electric field in the vicinity of the antennas, at frequency $f$, using the following integral equation [20]:

$$
Y_{12}(f)=\frac{1}{V_{1} V_{2}} \iiint_{V^{\prime}}\left(\mathbf{E}_{1}\left(r_{2} ; f\right) . \mathbf{J}_{2}\left(r_{2} ; f\right)\right) d V^{\prime},
$$

where $V_{1}$ and $V_{2}$ correspond to the excited voltage of each antenna, $\mathbf{E}_{1}$ is the strength of the radiated electric field of antenna 1 to the region $r_{2}$ of antenna 2 within a three dimensional volume $V^{\prime}$. This results in a current distribution, $\mathbf{J}_{2}$, at antenna 2 based on the infinitesimal dipole model.
Similarly, the electric field from antenna 2 causes a current distribution at antenna 1 . When both antennas are radiating simultaneously, the coupled current density to the opposite element and ground plane as well as each antenna's driven current will superimpose over each other thus substantially altering the current distribution of the whole UT, which will be dependent on the relative phase between elements.

Based on the findings in [21], an antenna's current distribution will directly impact the resulting tangential magnetic field $H_{\text {tp }}$ and penetrating electric field $E_{\mathrm{p}}$ into the surface of the head, where brain tissue has high dielectric constant, $\epsilon_{\mathrm{r}}$ :

$$
E_{\mathrm{p}}=\left(\frac{\mu_{0}}{\epsilon_{0} \epsilon_{\mathrm{r}}}\right) H_{\mathrm{tp}}
$$

where $\epsilon_{0}$ and $\mu_{0}$ are the permittivity and permiability of free space. Here the three principles come together where each antenna element receives less input power than a single element, thus $E_{\mathrm{p} 1}$ and $E_{\mathrm{p} 2}$ penetrating from each element are lower. However, they superimpose together to form a total field $E_{\mathrm{pT}}$, which is dependent on the relative phase between elements. Where there is high mutual coupling between elements, the change to current distribution can further reduce $E_{\mathrm{pT}}$ and hence SAR [22]:

$$
\mathrm{SAR}=\frac{\rho \times\left|E_{\mathrm{pT}}\right|^{2}}{M_{d}} \quad(\mathrm{~W} / \mathrm{kg}) .
$$

computed using density $M_{d}$ and conductivity $\rho$.

The SAR must comply with national and international EMF exposure limits given as $1.6 \mathrm{~W} / \mathrm{kg}$ averaged over $1 \mathrm{~g}$ of body tissue from the Federal Communications Commission (FCC) [23] and up to $2.0 \mathrm{~W} / \mathrm{kg}$ averaged over $10 \mathrm{~g}$ of body tissue from the International Commission on Non-ionizing Radiation Protection (ICNIRP) [1]. These values are based on the maximum possible transmit power from the UT [24]. The next two sections detail extensive simulations to analyse the effect of the three principles established here on SAR reduction well within the required limits.

\section{Coupling Manipulation Simulation Campaign}

A PIFA as a candidate antenna illustrated in Fig. 1 is selected in this study that utilizes the ground plane and has also been used in previous studies to reduce SAR [13] as well as allowing for suitably high coupling between elements. A ground plane size of length $L_{\mathrm{g}}=138 \mathrm{~mm}$, width $W_{\mathrm{g}}=68$ $\mathrm{mm}$ was chosen with thickness $t=0.8 \mathrm{~mm}$ following the dimensions of a typical smartphone. The PIFA elements taken from [25] have a fixed width $W_{\mathrm{p}}=10 \mathrm{~mm}$ while the length $L_{\mathrm{p}}$ is dependent on the element separation $d$ in order that $S_{11}$ (equal to $S_{22}$ ) is minimum at $2.4 \mathrm{GHz}$. Discrete values of $d$ between 7 and $62 \mathrm{~mm}$ and corresponding values of $L_{\mathrm{p}}$ are shown in Table I. Each PIFA element is connected to the ground plane by using a connecting pin and a feeding port (as it is shown in the right-hand side of Fig. 1) and $H_{\mathrm{g}}=6 \mathrm{~mm}$ is the height of each PIFA element above the ground plane. The values of both $S_{11}$ and $S_{21}$ (equal to $S_{12}$ ) are plotted in Fig. 2 for three use cases: 1) free space; 2) when using a sphere 


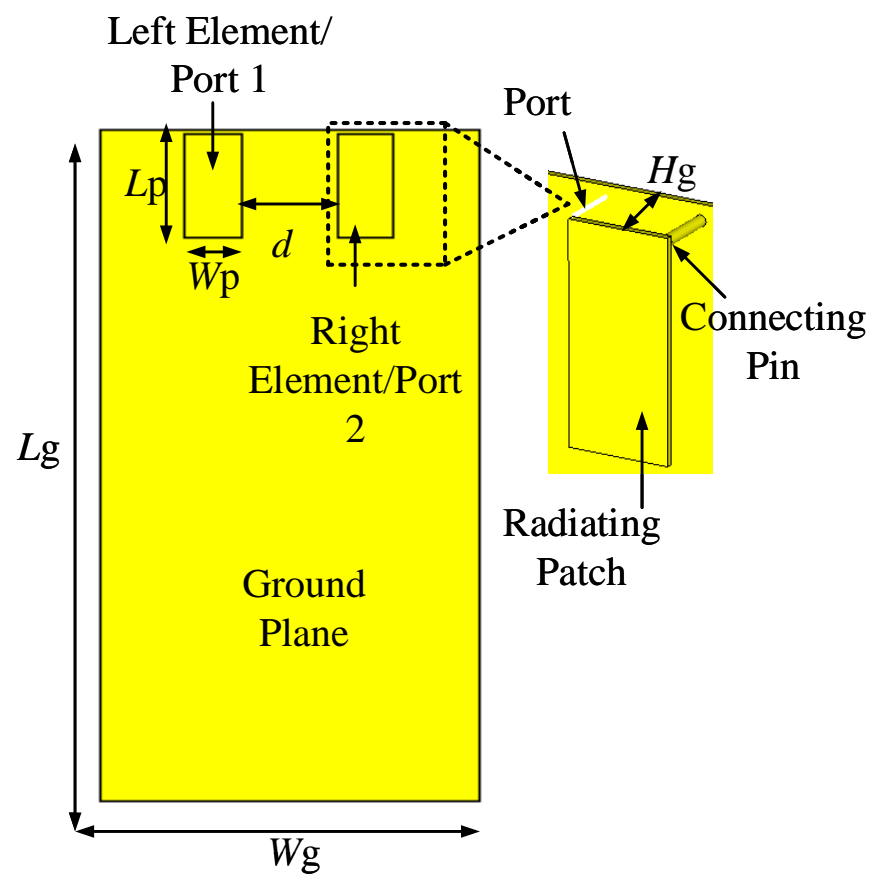

Fig. 1. An illustration of a classic PIFA arranged in a $2 \times 2$ MIMO configuration.

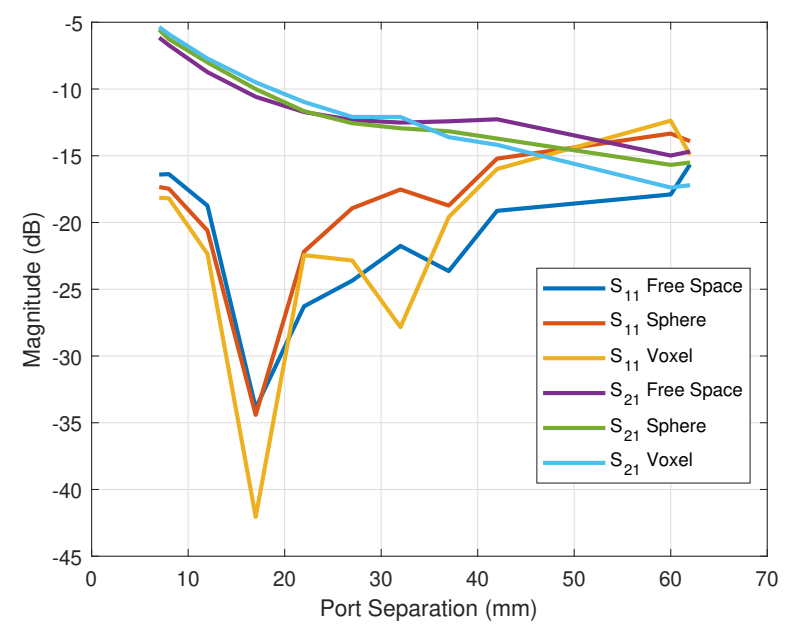

Fig. 2. The simulated $S_{11} \& S_{21}$ of a PIFA arranged in a $2 \times 2$ MIMO configuration, in the free space, aligned with the homogeneous spherical, and the heterogeneous voxel, head models, by varying the value of $d$ at a fixed resonance frequency of $2.4 \mathrm{GHz}$.

head homogeneous model and 3) when using a more realistic voxel (Donna: available in CST Voxel family) hetrogeneous model illustrated in Fig. 3. The results in Fig. 2 show that $S_{11}$ is sufficiently low below $-10 \mathrm{~dB}$ regardless of $d$ while $S_{21}$ decreases with an increase in $d$ as expected.

The simulation setup in Fig. 3 is based on IEEE recommendations [26], where the default handset orientation relative to the head is based on the cheek position for the voxel model. The center of the top of the ground plane, corresponding to the earpiece is also positioned in line with the ear. For the solid sphere model (conductivity $1.42 \mathrm{~S} / \mathrm{m}$ and dielectric
TABLE I

AdJusted VALUES OF $L_{\mathrm{P}}$ FOR EACH $d$.

\begin{tabular}{|l|l||l|l||l|l|}
\hline \hline$d(\mathrm{~mm})$ & $L_{\mathrm{p}}(\mathrm{mm})$ & $d(\mathrm{~mm})$ & $L_{\mathrm{p}}(\mathrm{mm})$ & $d(\mathrm{~mm})$ & $L_{\mathrm{p}}(\mathrm{mm})$ \\
\hline 7 & 21.1 & 22 & 21.38 & 42 & 20.80 \\
\hline 8 & 21.05 & 27 & 21.25 & 47 & 20.30 \\
\hline 12 & 21 & 32 & 21.25 & 60 & 20.90 \\
\hline 17 & 21.22 & 37 & 20.80 & 62 & 20.90 \\
\hline \hline
\end{tabular}
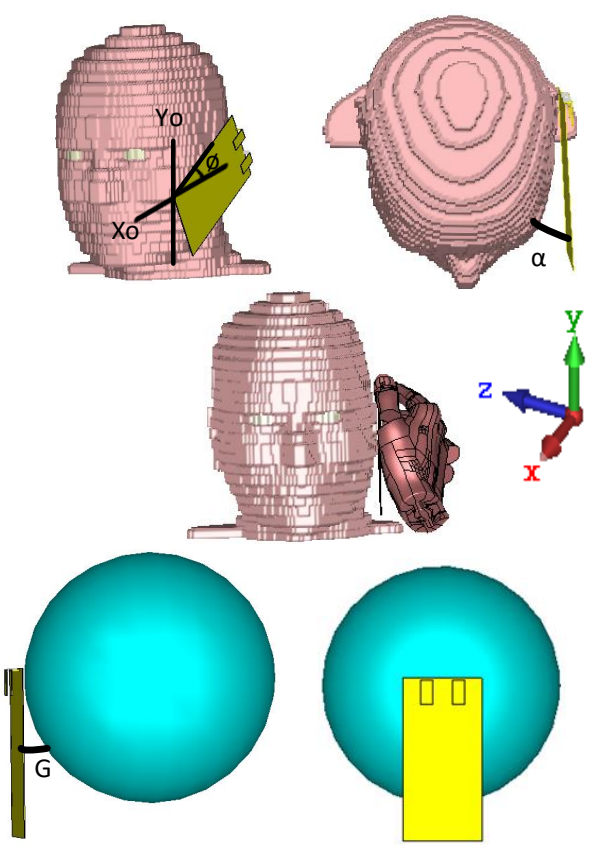

Fig. 3. The alignment of UT with the homogeneous spherical and heterogeneous voxel head models used for SAR analysis and following the IEEE recommendations.

constant 39.9), the ground plane is at a tangent with the handset earpiece aligned with the center. The gap $G$ between the voxel/sphere and the UT is set as $5 \mathrm{~mm}$, which is in line with industrial standards. For both cases, the IEEE/IEC 627041 method [27] is used to calculate the maximum averaged SAR (for $1 \mathrm{~g}$ of body mass) value by using the maximum long term evolution (LTE) uplink transmit power of $23 \mathrm{dBm}$, which is equally distributed between the two PIFA elements. The effect of hand grip shown in Fig. 3 is also analyzed later in Section V. Moreover, $\alpha$ indicates the tilt angle of the handset away from the cheek, which is assumed to be zero except when its effect is analyzed in Section V. The inclination angle $\phi$ is set to $60^{\circ}$.

Fig. 4 depicts the variations of the total efficiency using the blue curves as well as individual port efficiencies as a function of $d$. This is considered with the handset in free space, as well as in the presence of the sphere and voxel models. Note that both ports have equal efficiency in free space and, hence, only the efficiency of a single port is plotted. The total efficiency for both ports begins to drop substantially when $d$ is less than $17 \mathrm{~mm}$, which corresponds to less than $0.14 \lambda$, with $S_{21}$ from -9 to $-11 \mathrm{~dB}$ (taken from Fig. 2). The orange curves show the optimized SAR as a function of $d$ using the sphere and voxel models. Note that for each value of $d$ selected, the 


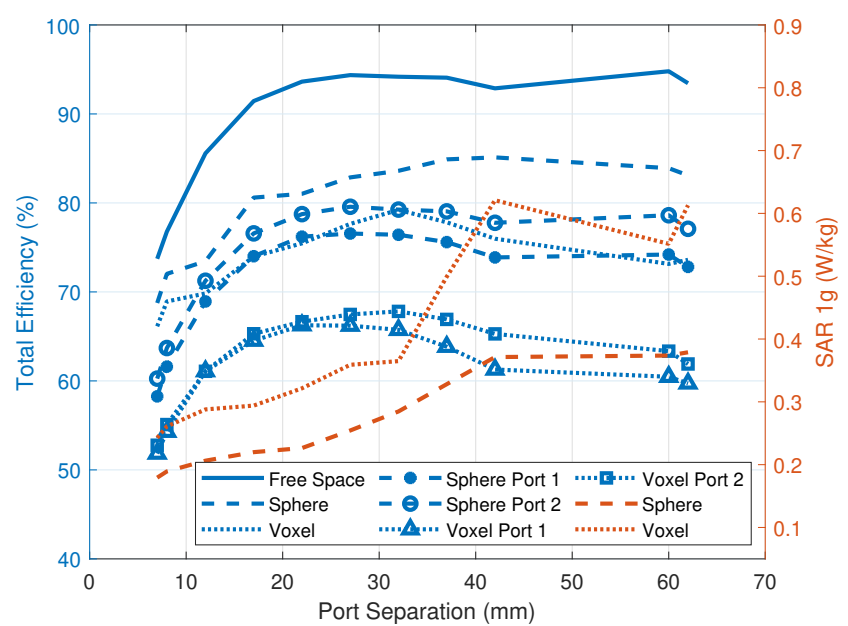

Fig. 4. Variation of the total efficiency in free space, variation of the total efficiency, and the SAR for a homogeneous sphere having a fixed phase of $0^{\circ}$ and heterogeneous voxel head models having a fixed phase of $30^{\circ}$ (when $d$ is varied from 7 to $32 \mathrm{~mm}$ ) and $0^{\circ}$ (when $d$ is varied from 37 to $62 \mathrm{~mm}$ ). Note that the selection of phase angles for sphere and voxel head models correspond to the minimum value of the SAR.

phase between elements is set corresponding to the lowest SAR, which is $30^{\circ}$ for $d=7$ to $32 \mathrm{~mm}$ and $0^{\circ}$ for $d=37$ to $62 \mathrm{~mm}$. The corresponding efficiency values also use these phase angles. Results indicate that the SAR can be drastically reduced by half when $d=17 \mathrm{~mm}$ compared with the maximum spacing at $62 \mathrm{~mm}$ that can be assumed to be a conventional MIMO antenna setup. Furthermore, efficiency is maintained at $17 \mathrm{~mm}$ comparable with that at $62 \mathrm{~mm}$. Efficiency drops substantially where $d<17 \mathrm{~mm}$ while further reduction in SAR is negligible.

Fig. 5 complements the results of Fig. 4 by illustrating the variations of the total antenna efficiency and SAR again with blue and orange curves as a function of the relative phase between the two antenna elements, or ports, when $d$ is fixed at either $17 \mathrm{~mm}$ or $62 \mathrm{~mm}$, or when only one port is used. It can clearly be seen that for both sphere and voxel models, the SAR changes dramatically with the relative phase, as it has been observed in [14]. As in Fig. 4, the SAR is lower for $d=17 \mathrm{~mm}$ than $d=62 \mathrm{~mm}$; this further indicates that the increased coupling between the antenna elements helps to reduce the SAR to a minimal value of $0.29 \mathrm{~W} / \mathrm{kg}$ and 0.21 $\mathrm{W} / \mathrm{kg}$ in the case of the voxel and sphere models, respectively, at a phase angle of $30^{\circ}$. At approximately $180^{\circ}$, the SAR reaches a maximum value of $1.4 \mathrm{~W} / \mathrm{kg}$ and $0.81 \mathrm{~W} / \mathrm{kg}$ for the voxel and sphere when $d=17 \mathrm{~mm}$. It is worth noting that the maxima (and minima) of the voxel and sphere models occur at the same phase angles and therefore the composition of the head tissues do not affect this. Furthermore, the maximum SAR of $1.4 \mathrm{~W} / \mathrm{kg}$ (for the voxel model) is less than the SAR of the individual antenna element when positioned at the right or left of a handset as the same transmit power is split into the two elements. The right and left elements are labelled in Fig. 1 and the SAR is different for each of them as they are positioned differently with respect to the head tissues. Though impractical, the SAR from a single PIFA in the center of the

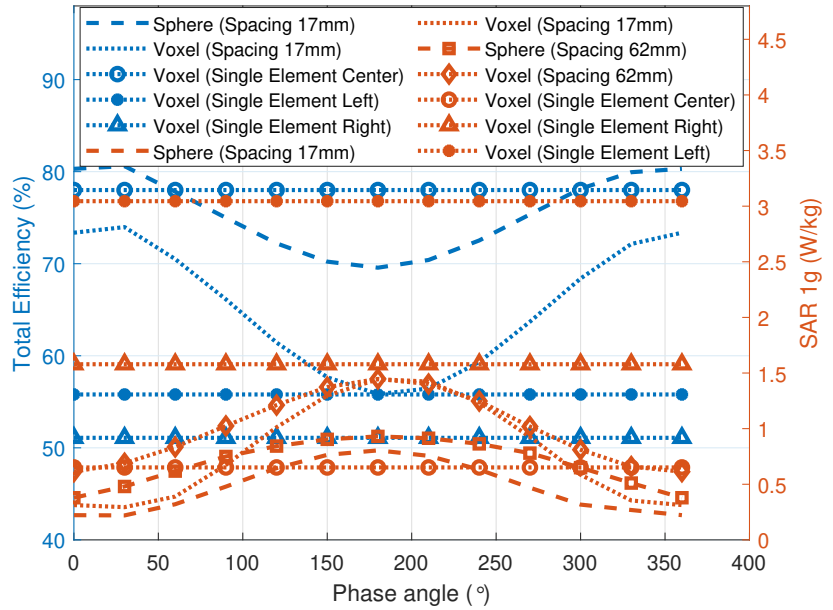

Fig. 5. The variation of SAR, and the total efficiency by varying the phase difference between antenna elements, as well as different single element configurations of PIFA (center, right and left), aligned with the homogeneous spherical and heterogeneous voxel head models.

handset, where the earpiece would be, is also plotted due to higher absorption by the ear but the voxel result for $17 \mathrm{~mm}$ spacing at $30^{\circ}$ still reduces SAR by $50 \%$, while compared with a right and left hand element, the reduction is $81 \%$ and $90 \%$ respectively. Therefore, the center spaced antenna elements are the best option to reduce the SAR in talk position. Comparing the voxel result at $30^{\circ}$ for $17 \mathrm{~mm}$ and $62 \mathrm{~mm}$ spacing, the reduction is also $50 \%$ as was found from Fig. 4, which justifies using the increased coupling while efficiency is maintained. Therefore the antennas are radiating away from the head as opposed to emitting heat.

\section{Surface CuRrent \& SAR ANALysis}

Having established the optimum phase angle for minimum SAR this section draws upon analysing the three physical principles established in section II. The following PIFA configurations are analysed with current distributions in Fig. 6, and SAR analyses in Figs. 7 (voxel) and 8 (sphere):

- A single element PIFA placed at the center, left and right of the ground plane in Figs. 6 (a), (b) and (c) and Figs. 7 (a), (b) and (c) respectively.

- A low coupling level case $(d=62 \mathrm{~mm})$ with phase angles of $0^{\circ}$ and $180^{\circ}$ (corresponding to the minimum and maximum SAR values as a function of the phase in Fig. 5) in Figs. 6 (d) and (e), Figs. 7 (d) and (e) and Figs. 8 (a) and (b), respectively.

- A high coupling level case $(d=17 \mathrm{~mm})$ with phase angles of $30^{\circ}$ and $180^{\circ}$ (corresponding to the minimum and maximum SAR values as a function of the phase in Fig. 5) in Figs. 6 (f) and (g), Figs. 7 (f) and (g) and Figs. 8 (c) and (d), respectively.

Fig. 6 is displayed with surface currents set to a maximum peak colorbar level of $15 \mathrm{~A} / \mathrm{m}$ for purposes of clarity while the maximum actual current is stated on each sub figure. The current distribution is shown on both the front (top part of each sub figure) and rear (bottom part of each sub figure) of 


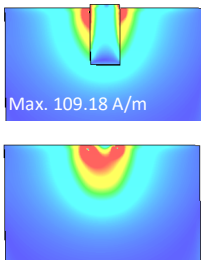

(a)

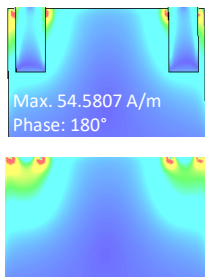

(e)
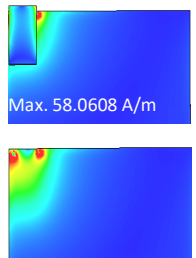

(b)

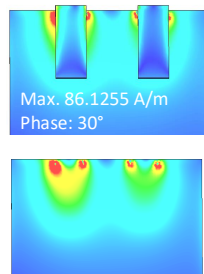

(f)
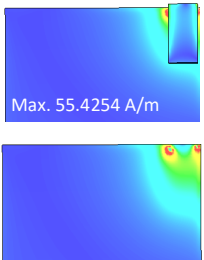

(c)

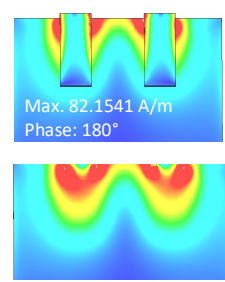

(g)
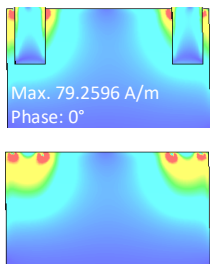

(d)

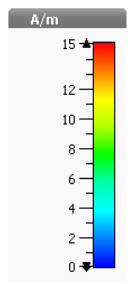

Fig. 6. A surface current distribution comparison of different PIFA arrangements aligned with the heterogeneous voxel model; (a) Single element PIFA at the center of the ground plane, (b) Single element PIFA on the left of the ground plane, (c) Single element PIFA on the right of the ground plane, (d) Two element low coupling PIFA configuration with $0^{\circ}$ phase difference, (e) Two element low coupling PIFA configuration with $180^{\circ}$ phase difference, (f) Two element high coupling PIFA configuration with $30^{\circ}$ phase difference, (g) Two element high coupling PIFA configuration with $180^{\circ}$ phase difference Note: Maximum current in colorbar is limited at $15 \mathrm{~A} / \mathrm{m}$ but the actual maximum current is stated on each sub figure.

the UT in the region of the PIFA elements. Figs. 7 and 8 are cross sectioned at the level of the earpiece and for clarity the colorbar has been set to a limit of $0.2 \mathrm{~W} / \mathrm{kg}$.

Figs. 6 (a) to (c) show that each radiating element has a strong current formed by the feed and the ground plane has a strong current close to the PIFA. A wider spread of current is found on the ground plane when the PIFA is in the center, as it interacts differently with the ground plane while the ear also reduces the SAR through to the head when comparing Figs. 7 (a) to (c). Note that the left antenna is closer to the head, hence creating a higher SAR than the right antenna (as shown in Fig. 5).

The low coupling case in Fig. 8 (a) shows that where the phase is set for the lowest SAR, the penetrating fields add in phase at the mid point between the two elements, but out of phase at each element. Setting the relative phase from $0^{\circ}$ to $180^{\circ}$ in Fig. 8 (b) causes the inverse effect as expected. Therefore by splitting the power evenly between the two elements, the lowest SAR occurs with the phase set so that the penetrating fields with the lowest magnitude add together constructively. By increasing the coupling with closer elements in Figs. 8 (c) (d), the same principle of penetrating fields adding together in and out of phase occurs but additionally there is substantial change to the current distribution especially on the ground plane of the UT as seen in Figs. 6 (f) and (g). This will create substantial change to the total tangential magnetic field $H_{\mathrm{tpT}}$ and penetrating electric field $E_{\mathrm{pT}}$, which minimises with a phase of $30^{\circ}$. This subsequently enables all three principles discussed in section II to work in harmony to minimise the SAR.

The sphere model is a useful way to analyse how the physical principles work to obtain the minimum SAR with

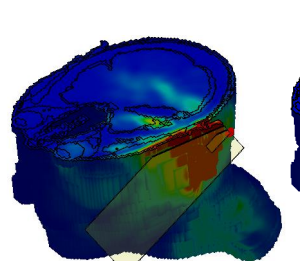

(a) Center

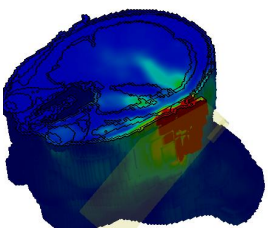

(d) Low Coupling Phase: $0^{\circ}$

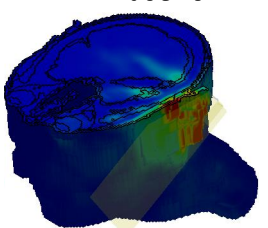

(f) High Coupling Phase: $30^{\circ}$

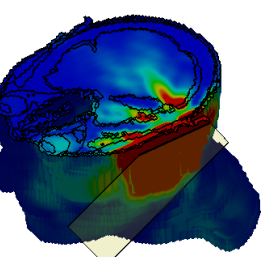

(b) Left

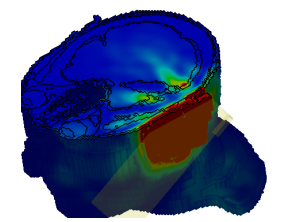

(e) Low Coupling Phase: $180^{\circ}$

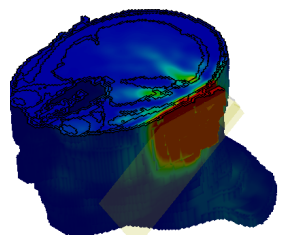

(g) High Coupling Phase: $180^{\circ}$
Fig. 7. A SAR cross section comparison of different PIFA arrangements aligned with heterogeneous voxel model; (a) Single element PIFA at center of the ground plane, (b) Single element PIFA at left of the ground plane, (c) Single element PIFA at right of the ground plane, (d) Two element low coupling PIFA configuration with $0^{\circ}$ phase difference, (e) Two element low coupling PIFA configuration with $180^{\circ}$ phase difference, (f) Two element high coupling PIFA configuration with $30^{\circ}$ phase difference, (g) Two element high coupling PIFA configuration with $180^{\circ}$ phase difference.

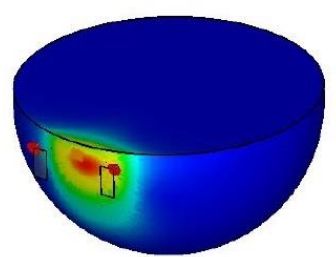

(a) Low Coupling Phase $0^{\circ}$

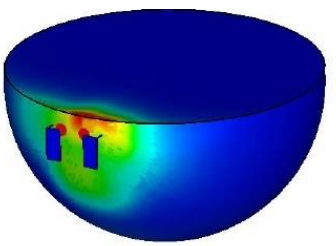

(c) High Coupling Phase $30^{\circ}$

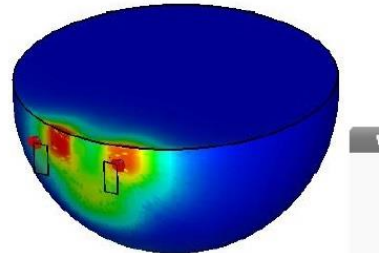

(b) Low Coupling Phase $180^{\circ}$

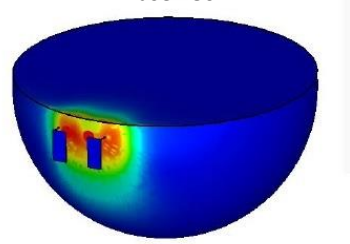

(d) High Coupling Phase $180^{\circ}$
Fig. 8. A SAR cross section comparison of different PIFA arrangements aligned with homogeneous sphere model; (a) Two element low coupling with $0^{\circ}$ phase difference, (b) Two element low coupling with $180^{\circ}$ phase difference, (c) Two element high coupling with $30^{\circ}$ phase difference, (d) Two element high coupling with $180^{\circ}$ phase difference.

a spacing $d$ of $17 \mathrm{~mm}$ and a relative phase angle of $30^{\circ}$ for the PIFA elements. The same effect occurs in a voxel head though with less clarity as shown in Figs. 7 (d) to (g) because the presence of the ear causes the penetrated fields to distribute unevenly. Nonetheless the SAR in the ear is shown to be 


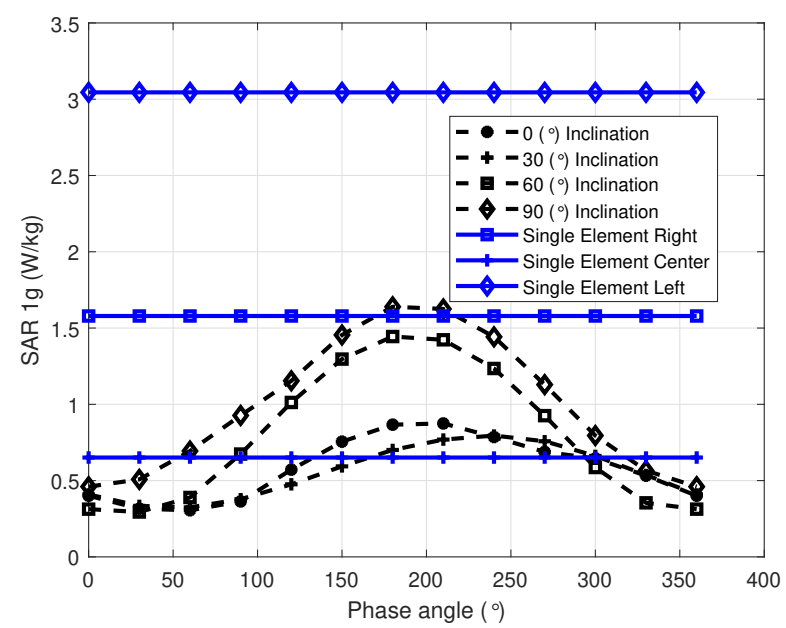

Fig. 9. The variation of SAR vs phase angle between elements with fixed element spacing of $17 \mathrm{~mm}$ and different inclination angles compared with single elements at $60^{\circ}$ inclination.

minimal in Fig. 7 (f). A final noteworthy point is that a substantial ground plane is also required as with PIFAs in order to have enough conducting volume to substantially change the current distribution when the elements are closely coupled. This could not be achieved with smaller conducting structures such as wire antennas.

\section{Resilience to Different Head Use Cases}

Up to this point, only the fixed position of a $60^{\circ}$ inclination angle has been considered. However, it is also important to analyze the variations of the SAR when the handset deviates from this position, notably due to an offset of the earpiece relative to the ear hole in talk position and/or a change in inclination or tilt angle. Furthermore, the effects of the hand grip in a typical use case are also important to take into account. These effects on the SAR are analyzed with the following simulation settings when $d=17 \mathrm{~mm}$ :

- The inclination angle $\phi$ is set to $0^{\circ}, 30^{\circ}, 60^{\circ}$, or $90^{\circ}$ to reflect the range of angles with which the handset may be held.

- The tilt angle $\alpha$ is increased to $15^{\circ}$, following the IEEE recommendations [26].

- The UT is offset by moving the handset along the $x$ or $y$ directions. More specifically, the value of $X_{0}$ or $Y_{0}$ in Fig. 3 is varied by $\pm 10 \mathrm{~mm}$ to achieve four different offsets, i.e. $10 \mathrm{~mm}$ upward, $10 \mathrm{~mm}$ downward, $10 \mathrm{~mm}$ right, and $10 \mathrm{~mm}$ left to reflect the maximum expected position offset of the handset with regard to the earpiece aligning with the ear hole.

Figs. 9 and 10 show the variations of the SAR as a function of the phase angle for the various aforementioned configurations. With regard to the inclination angle, the minimum SAR is maintained at a phase angle of $30^{\circ}$ except for the instance where $\phi$ is $90^{\circ}$. Even in this instance however, it increases only up to $0.5 \mathrm{~W} / \mathrm{kg}$, which is still substantially lower by a factor of three or more when comparing to a single element on the left

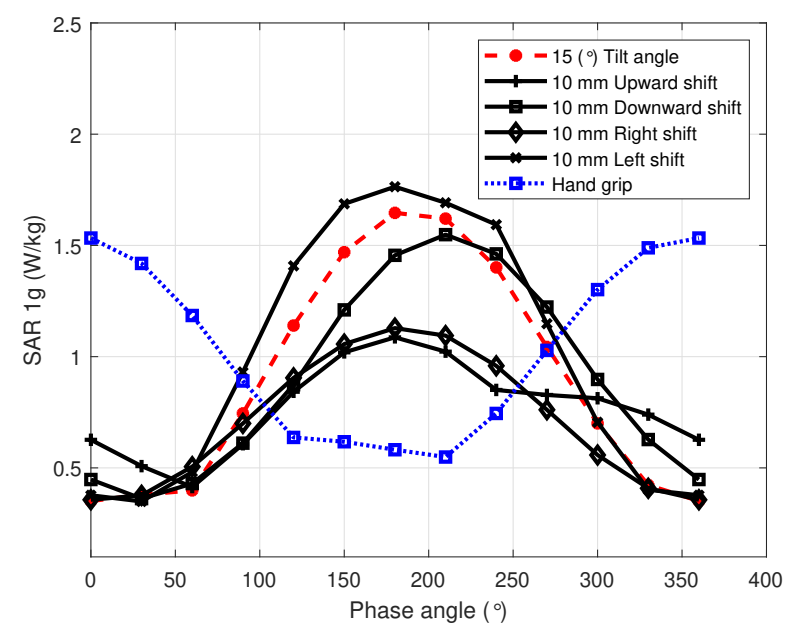

Fig. 10. The variation of SAR vs phase angle between elements with fixed element spacing of $17 \mathrm{~mm}$ and $60^{\circ}$ inclination angle with a change in tilt angle to $15^{\circ}$ and spatial earpiece offset in the upward, downward, left and right directions.

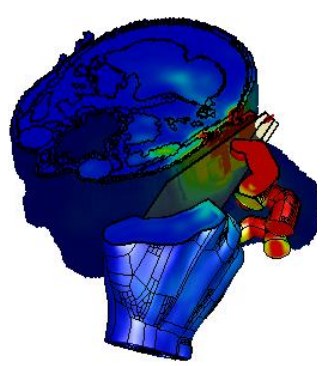

(a) High Coupling Phase: $210^{\circ}$

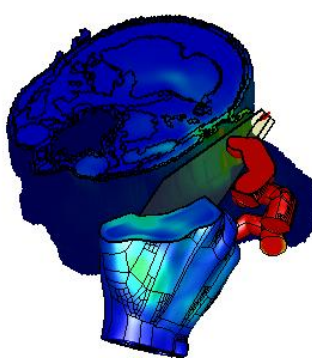

(b) High Coupling Phase: $0^{\circ}$

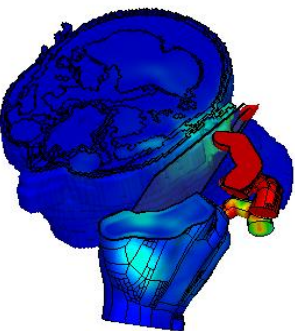

(c) High Coupling Phase: $30^{\circ}$

Fig. 11. A SAR penetration comparison aligned with voxel head model using a hand grip (a) A high coupling configuration with $210^{\circ}$ phase difference (b) A high coupling configuration with $0^{\circ}$ phase difference (c) A high coupling configuration with $30^{\circ}$ phase difference.

or right of the handset. With regard to UT offset, the minimum SAR is still maintained at a phase angle of $30^{\circ}$ except for the case when $10 \mathrm{~mm}$ upward shift is applied. However, its SAR is still only $0.5 \mathrm{~W} / \mathrm{kg}$ equal to the scenario for $\phi=90^{\circ}$.

With regard to the effect of hand grip, a worst case scenario illustrated in Fig. 3 is applied here whereby the fingers cover the antennas on the handset. Fig. 10 shows that the minimum SAR is not maintained anymore at a phase angle of $30^{\circ}$ and the curve is almost inverted. Indeed, the averaged SAR is calculated in this instance with the inclusion of the hand and Figs. 11 (a) and (b), clearly indicate that substantial absorption 


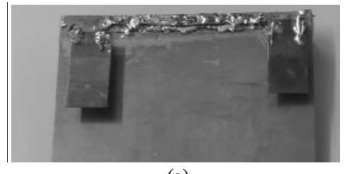

(a)

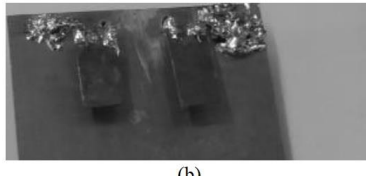

(b)

Fig. 12. Prototype of the UT with (a) $d=17 \mathrm{~mm}$ and (b) $d=62 \mathrm{~mm}$.

goes directly into the hand. Figs. 11 (a) and (b) depict the SAR penetration for a relative phase angle of $210^{\circ}$ (minimum SAR in Fig. 10) and $0^{\circ}$ (maximum SAR in Fig. 10). It is clear that where the averaged SAR is lowest at $210^{\circ}$ (according to Fig. 10 ), there is actually more SAR in the head for this relative phase than for $0^{\circ}$, according to Fig. 11. Therefore it can be inferred that the SAR in the head is still minimum with a phase angle of $30^{\circ}$, which is confirmed by Fig. 11 (c) showing less than $0.08 \mathrm{~W} / \mathrm{kg}$ at any point in the head. Therefore, hand grip has no detrimental effect as far as minimizing SAR to the head is concerned.

Finally it is important to note that in this work, the power has been split equally between elements and is the optimum split due to where the two antennas are positioned. If an optimum position was found elsewhere on the UT this may result in a different power ratio being required.

\section{Mimo Performance AnAlysis in Data Mode}

Where the handset is used away from the head in what can be termed 'data mode' (which can be detected by a smartphone using its own accelerometer), it can reconfigure to use MIMO precoding instead of a fixed phase for low SAR against the head. However, the capacity may be reduced, as the mutual coupling should ideally be less than $-15 \mathrm{~dB}$ [25]. The correlation coefficient has a direct effect on the capacity [28] where for a $2 \times 2$ MIMO channel with a suitably high signal-to-noise ratio (SNR), the following expression can be used to determine the loss in capacity [29], in comparison with perfect MIMO antennas:

$$
\bar{C}_{\text {loss }}=-\log _{2}\left|\begin{array}{ll}
\rho_{1,1} & \rho_{1,2} \\
\rho_{2,1} & \rho_{2,2}
\end{array}\right|
$$

such that using S-parameters $\rho_{i, i}=\left(1-\left|S_{i, i}\right|^{2}+\left|S_{i, j}\right|^{2}\right)$ and $\rho_{i, j}=-\left(S_{i, i}^{*} S_{i, j}+S_{j, i}^{*} S_{j, j}\right), i$ and $j \leq 2$.

Prototypes for the PIFA with $d$ of $17 \mathrm{~mm}$ and $62 \mathrm{~mm}$ are illustrated in Fig. 12 and measurements of S-parameters are compared with simulations in Fig. 13. Results are in good agreement, though note that $\left|S_{21}\right|$ in the case of high coupling shows the measured result to be $-9 \mathrm{~dB}$ rather than $-11 \mathrm{~dB}$ in the simulated case. However, for such close spacing, there is coupling between the connectors that can be used to justify such difference. A further important metric for the closely spaced antennas is that the efficiency must not degrade and the comparisons of simulated efficiency are outlined in Table II at $2.4 \mathrm{GHz}$. These are validated by comparing the simulated and measured peak gains in the elevation plane normal to the ground plane. Results are within $1.6 \mathrm{~dB}$ difference owing to effects of the connectors and measurement cable but nonetheless they verify that efficiency is suitably maintained. It is clear

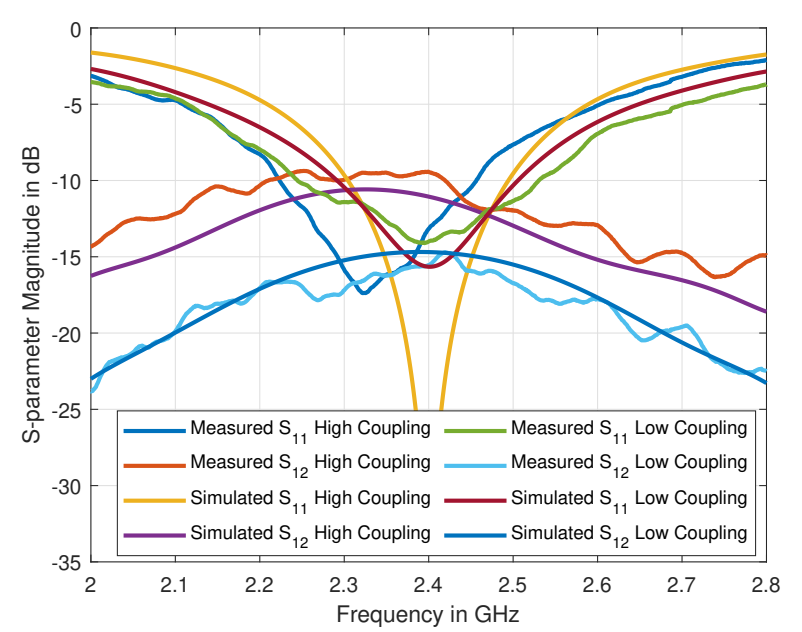

Fig. 13. Comparison of simulated and measured S-parameters for the UT with $d=17 \mathrm{~mm}$ (high) and $d=62 \mathrm{~mm}$ (low) coupling in free space.

TABLE II

COMPARISON OF UT METRICS WITH $d=17 \mathrm{MM}$ AND $d=62 \mathrm{MM}$ AT 2.4GHZ. Simulated (S) AND MEASURED (M) RESUlTS SHOWN.

\begin{tabular}{|l|l|l|l|l|}
\hline \hline $\begin{array}{l}d \\
(\mathrm{~mm})\end{array}$ & $\begin{array}{l}\text { Efficiency } \\
(\%)\end{array}$ & $\begin{array}{l}\text { Gain } \\
(\mathrm{dBi})\end{array}$ & $\begin{array}{l}S_{21} \\
(\mathrm{~dB})\end{array}$ & $\begin{array}{l}\bar{C}_{\text {loss }} \\
(\mathrm{bits} / \mathrm{s} / \mathrm{Hz})\end{array}$ \\
\hline 62 & $93.38(\mathrm{~S})$ & $5.60(\mathrm{~S})$ & $-15.00(\mathrm{~S})$ & $0.14(\mathrm{~S})$ \\
& & $4.04(\mathrm{M})$ & $-15.60(\mathrm{M})$ & $0.20(\mathrm{M})$ \\
\hline 17 & $91.55(\mathrm{~S})$ & $2.56(\mathrm{~S})$ & $-11.10(\mathrm{~S})$ & $0.23(\mathrm{~S})$ \\
& & $2.28(\mathrm{M})$ & $-9.40(\mathrm{M})$ & $0.41(\mathrm{M})$ \\
\hline \hline
\end{tabular}

that where $S_{21}$ is up to $-9 \mathrm{~dB}$ that a modest capacity loss will have to be accepted. Nonetheless this issue could be overcome such as by placing PIFA antennas with $d=17 \mathrm{~mm}$ at the the earpiece, while those with $d=62 \mathrm{~mm}$ at the bottom near the microphone and switching antennas according to the use case.

\section{CONCLUSION}

In this paper, a rigorous analysis has been carried out of how SAR to the human head can be reduced from a smartphone handset in talk position using two antenna elements by distributing the source power two ways and setting the relative phase between them to enable the penetrated fields to superimpose to form the lowest magnitude. Additionally, the coupling between them can be utilised when spaced close enough. SAR can be reduced by a factor of three or more compared to a single element when optimized. This has been shown to work consistently regardless of the head it is against or the user handling that is applied as long as the assumption is held that the earpiece is against the ear. When the device is away from the body, it is still suitable as a two element MIMO antenna while adaptive techniques could be adopted.

\section{REFERENCES}

[1] ICNIRP, "Guidelines for limiting exposure to electromagnetic fields (100 kHz to $300 \mathrm{GHz}$ )," Health Phys, vol. 118, p. 000-000, 2020.

[2] M. A. Imran, F. Héliot, and Y. A. Sambo, Low Electromagnetic Emission Wireless Network Technologies: $5 G$ and Beyond. Institution of Engineering and Technology, 2019. 
[3] G. S. . 38.151, "Nr; user equipment (ue) multiple input multiple output (mimo) over-the-air (ota) performance requirements," Release 17, 2020.

[4] I. P802.11, "Telecommunications and information exchange between systems local and metropolitan area networks, part 11: Wireless lan medium access control (mac) and physical layer (phy) specifications," Advanced technical ceramics. Monolithic ceramics. Gerneral and textural properties., 2020.

[5] W. Jiang, Y. Cui, B. Liu, W. Hu, and Y. Xi, "A Dual-Band MIMO Antenna With Enhanced Isolation for 5G Smartphone Applications," IEEE Access, vol. 7, pp. 112554-112563, 2019.

[6] X.-T. Yuan, W. He, K.-D. Hong, C.-Z. Han, Z. Chen, and T. Yuan, "Ultra-Wideband MIMO Antenna System With High Element-Isolation for 5G Smartphone Application," IEEE Access, vol. 8, pp. 56281-56289, 2020.

[7] J. Wang, O. Fujiwara, and T. Takagi, "Effects of ferrite sheet attachment to portable telephone in reducing electromagnetic absorption in human head," IEEE International Symposium on Electromagnetic Compatability, vol. 2, pp. 822-825, Aug 1999.

[8] M. I. Kitra, C. J. Panagamuwa, P. McEvoy, J. C. Vardaxoglou, and J. R. James, "Low SAR Ferrite Handset Antenna Design," IEEE Transactions on Antennas and Propagation, vol. 55, pp. 1155-1164, April 2007.

[9] M. F. Abedin and M. Ali, "Modifying the ground plane and its effect on planar inverted-F antennas (PIFAs) for mobile phone handsets," IEEE Antennas and Wireless Propagation Letters, vol. 2, pp. 226-229, 2003.

[10] H. H. Zhang, G. G. Yu, Y. Liu, Y. X. Fang, G. Shi, and S. Wang, "Design of low-sar mobile phone antenna: Theory and applications," IEEE Transactions on Antennas and Propagation In Press, pp. 1-1, 2021.

[11] J. Hwang and F. Chen, "Reduction of the Peak SAR in the Human Head With Metamaterials," IEEE Transactions on Antennas and Propagation, vol. 54, pp. 3763-3770, Dec 2006.

[12] R. Gómez-Villanueva, H. Jardón-Aguilar, and R. L. y Miranda, "State of the art methods for low sar antenna implementation," Proceedings of the Fourth European Conference on Antennas and Propagation, pp. 1-4, April 2010.

[13] K.-C. Chim, K. C. Chan, and R. D. Murch, "Investigating the impact of smart antennas on SAR," IEEE Transactions on Antennas and Propagation, vol. 52, no. 5, pp. 1370-1374, 2004.

[14] B. M. Hochwald, D. J. Love, S. Yan, and J. Jin, "SAR codes," in Information Theory and Applications Workshop (ITA), 2013, pp. 1-9, IEEE, 2013.

[15] M. A. Baldauf, J. A. Pontes, J. Timmermann, and W. Wiesbeck, "Mobile MIMO phones and their human exposure to electromagnetic fields," in Electromagnetics in Advanced Applications, 2007. ICEAA 2007. International Conference on, pp. 9-12, IEEE, 2007.

[16] H. Li, A. Tsiaras, and B. K. Lau, "Analysis and estimation of MIMOSAR for multi-antenna mobile handsets," IEEE Transactions on Antennas and Propagation, vol. 65, no. 3, pp. 1522-1527, 2017.

[17] K. Zhao, S. Zhang, Z. Ying, T. Bolin, and S. He, "SAR Study of Different MIMO Antenna Designs for LTE Application in Smart Mobile Handsets," IEEE Transactions on Antennas and Propagation, vol. 61 , pp. 3270-3279, June 2013.

[18] F. Héliot, M. A. Jamshed, and T. W. Brown, "Exposure Modelling and Minimization for Multi-antenna Communication Systems," in 2020 IEEE 91st Vehicular Technology Conference (VTC2020-Spring), pp. 16, IEEE, 2020.

[19] C. A. Balanis, Antenna theory: analysis and design. John wiley \& sons, 2016.

[20] Y.-D. Kim, S.-J. Yang, Y.-S. Kang, I.-J. Hwang, and J.-W. Yu, "Mutual Admittance of Two Arbitrary Antennas in Nonplanar Skew Positions Based on Infinitesimal Dipole Modeling," IEEE Transactions on Antennas and Propagation, vol. 67, no. 11, pp. 6705-6713, 2019.

[21] H. Wang, "Analysis of electromagnetic energy absorption in the human body for mobile terminals," IEEE Open Journal of Antennas and Propagation, vol. 1, pp. 113-117, 2020.

[22] M. A. Jamshed, F. Heliot, and T. Brown, "A Survey on Electromagnetic Risk Assessment and Evaluation Mechanism for Future Wireless Communication Systems," IEEE Journal of Electromagnetics, RF and Microwaves in Medicine and Biology, 2019.

[23] Radiofrequency Electromagnetic Fields, "Evaluating compliance with FCC guidelines for human exposure to radio frequency electromagnetic fields," OET Bull, vol. 65, pp. 1-53, 1997.

[24] F. C. Commission et al., "Specific absorption rate (SAR) for cell phones: what it means for you," 2014.

[25] A. Chebihi, C. Luxey, A. Diallo, P. Le Thuc, and R. Staraj, "A novel isolation technique for closely spaced pifas for umts mobile phones,"
IEEE Antennas and Wireless Propagation Letters, vol. 7, pp. 665-668, 2008.

[26] "IEEE Recommended Practice for Determining the Peak SpatialAverage Specific Absorption Rate (SAR) in the Human Head from Wireless Communications Devices: Measurement Techniques," IEEE Std 1528-2013 (Revision of IEEE Std 1528-2003), pp. 1-246, Sep. 2013.

[27] "IEC/IEEE International Standard - Determining the peak spatialaverage specific absorption rate (SAR) in the human body from wireless communications devices, $30 \mathrm{MHz}$ to $6 \mathrm{GHz}$ - Part 1: General requirements for using the finite-difference time-domain (FDTD) method for SAR calculations," IEC/IEEE 62704-1:2017, pp. 1-86, Oct 2017.

[28] S. H. Chae, S.-k. Oh, and S.-O. Park, "Analysis of mutual coupling, correlations, and TARC in WiBro MIMO array antenna," IEEE Antennas and Wireless Propagation Letters, vol. 6, pp. 122-125, 2007.

[29] H. Shin and J. H. Lee, "Capacity of multiple-antenna fading channels: Spatial fading correlation, double scattering, and keyhole," IEEE Transactions on Information Theory, vol. 49, no. 10, pp. 2636-2647, 2003.

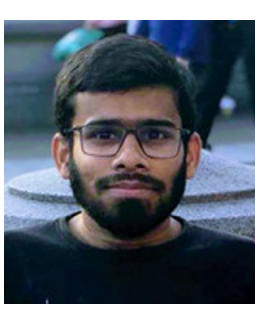

Muhammad Ali Jamshed (S'19-M'21) received the B.Sc. degree in electrical engineering from COMSATS University, Islamabad, Pakistan, in 2013 and the M.Sc. degree in Wireless communications from the Institute of Space Technology, Islamabad Pakistan, in 2016, and a Ph.D. degree from the University of Surrey, Guildford, U.K, in 2021. He was nominated for Departmental Prize for Excellence in Research in 2019 at the University of Surrey. He served briefly as Wireless Research Engineer at BriteYellow Ltd, UK, and then moved to James Watt School of Engineering, University of Glasgow, as a Research Assistant. His main research interests include EMF exposure reduction, low SAR antennas for mobile handsets, Backscatter communication, and wireless sensor networks.

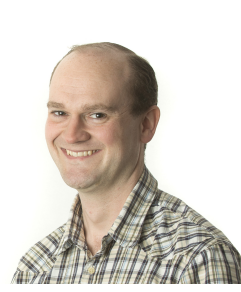

Tim W. C. Brown (S'00-M'04) received the B.Eng. degree in electronic engineering from the University of Surrey, Surrey, U.K., in 1999, and the Ph.D. degree in antenna diversity for mobile terminals from the Institute for Communications Systems, University of Surrey, Guildford, U.K., in 2004. Since 2004, he has been involved in antennas, propagation, and radio frequency (RF) engineering. From 2004 to 2006, he was a Post-Doctoral Researcher from Aalborg University, Aalborg, Denmark. He is currently an Associate Professor in RF, antennas, and propagation with the University of Surrey. His current research interests include intelligent antennas for mobile and satellite communications, propagation modeling from a few megahertz to tens of gigahertz, MIMO, UWB, and smart RFID.

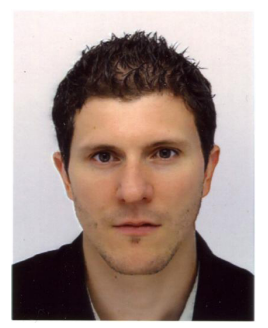

Fabien Héliot (S'05-M'07) received the M.Sc. degree in telecommunications from the Institut Supérieur de l'Electronique et du Numerique (ISEN), Toulon, France, in 2002 and the Ph.D. degree in mobile telecommunications from King's College London, London, U.K., in 2006. He is currently a Lecturer at the Institute for Communication Systems (ICS) of the University of Surrey, formerly known as CCSR. He has been actively involved in European Commission (EC) funded projects such as FIREWORKS, ROCKET, SMART-Net, LEXNET projects and in the award-winning EARTH project. He is currently involved in the 5GIC project, a UK funded project on shaping the future of wireless communication. His research interests include energy efficiency, EM exposure reduction, cooperative communication, MIMO, and radio resource management. He received an Exemplary Reviewer Award from the IEEE COMMUNICATIONS LETTERS in 2011. 